\title{
REFLECTED BSDE'S WITH DISCONTINUOUS BARRIER AND TIME DELAYED GENERATORS
}

\author{
Monia KarouF ${ }^{1}$
}

\begin{abstract}
In this paper, we prove the existence and uniqueness result of the reflected BSDE with time delayed generator and right continuous and left limited barrier. A comparison theorem is also proved.
\end{abstract}

Mathematics Subject Classification. 60H10, 60H20, 34K12.

Received January 9, 2013. Revised October 9, 2013.

\section{INTRODUCTION}

Backward stochastic differential equations were firstly introduced by Bismut [2] in 1973 as equation for the adjoint process in the stochastic version of Pontryagin maximum principle. Pardoux and Peng [16] generalized the notion in 1990 and were the first ones to consider general BSDE's and to solve the question of existence and uniqueness in the non-linear case.

Since then, the theory of BSDE's has found further important applications and has become a powerful tool in many fields such as finance (see e.g. $[3,11]$ ), stochastic control, differential games (see e.g. [13,14]), partial differential equations (see e.g. $[1,15,17]$ ), obstacle problems, variational inequalities, and so on.

In [10], El-Karoui et al. introduced the notion of a reflected BSDE. Actually, it is a standard BSDE with an additional continuous increasing process in the equation to keep the solution above a given continuous process which represents an obstacle. Later, Cvitanic and Karatzas generalized in [3] the setting of [10] where they introduced BSDEs with two reflecting barriers. Hamadène [12] studied the case of a right-continuous with left limits barrier (càdlàg for short).

More recently, a new class of BSDE, called backward stochastic differential equation with time delay generators (delayed BSDEs for short), were investigated for the first time in [7], and in more depth in [8]. The dynamics of these BSDEs is given by

$$
Y(t)=\xi+\int_{t}^{T} g\left(s, Y_{s}, Z_{s}\right) \mathrm{d} s-\int_{t}^{T} Z(s) \mathrm{d} B(s), \quad t \in[0, T]
$$

where the generator $g$ at time $s$ depends in some measurable way on the past values of a solution $\left(Y_{s}, Z_{s}\right)=$ $(Y(s+u), Z(s+u))_{-T \leq u \leq 0}$. In $[7,8]$ existence and uniqueness questions are treated, and examples are given in

Keywords and phrases. Reflected backward stochastic differential equation, time delayed generator, comparison principle.

1 Université de Carthage, Unité de recherche, Analyse Stochastique et Modélisation Statistique, Tunisie.

Monia.Karouf@fsg.rnu.tn 
which multiple solutions or no solution at all exists. Further, some properties are studied, including comparison principle, measure solution and BMO martingales property for the control component $Z$.

These equations are a new research area concerning both theory and applications. Only so far in Delong [6], specific forms of time delayed BSDEs are derived and their explicit connections with pricing, hedging, management and insurance risks are given.

Dos Reis et al. [9] refine and extend the existence and uniqueness results obtained in Delong and Imkeller $([7,8])$. The authors provide sharper a priori estimates and show that the solution of a delayed BSDE is in $L^{p}$. Using these results, they give sufficient conditions for the variational differentiability of delayed forward BSDE. Then, they connect these derivatives to the Malliavin derivatives of such FBSDE via the usual representation formulas.

In a recent paper, Delong [5] shows that linear time-delayed BSDE associated with a bounded terminal value $\xi$ and a generator $g$ of a moving average type, i.e., $g$ depending on $\left(\frac{1}{t} \int_{0}^{t} Y(s) \mathrm{d} s, \frac{1}{t} \int_{0}^{t} Z(s) \mathrm{d} s\right)$, has an explicit solution. In the same paper, more insight into properties of solutions to time-delayed BSDEs is also given.

Zhou and Ren [18] extend the set up of Delong-Imkeller [7] to the case of BSDEs with one continuous reflecting barrier. They show the existence and uniqueness of the solution when the coefficient $g$ is Lipschitz. The main objective of the present paper is to deal with reflected delayed BSDEs when the reflecting process is just càdlàg.

The paper is organized as follows: in Section 2, we introduce some preliminaries and notations. In Section 3 our main result is stated and proved. A comparison theorem of delayed RBSDEs with continuous barrier is given in the last section.

\section{Setting of the PRoblem}

The purpose of this section is to introduce some basic notations, which are needed throughout this paper.

Let $(\Omega, \mathcal{F}, \mathbb{P})$ be a complete probability space, and $B=\left(B_{t}\right)_{t \leq T}$ be a standard m-dimensional Brownian motion defined on the finite interval $[0, T], 0<T<+\infty$. Denote by $\left(\mathcal{F}_{t}^{0}:=\sigma\left\{B_{s}, s \leq t\right\}\right)_{t \leq T}$ the natural filtration of $B$ and $\left(\mathcal{F}_{t}\right)_{t \leq T}$ its completion with the $\mathbb{P}$-null sets of $\mathcal{F}$, therefore $\left(\mathcal{F}_{t}\right)_{t \leq T}$ satisfies the usual conditions, i.e. it is right continuous and complete. We define the following spaces:

- $\mathcal{P}$ the set of $\mathbb{R}$ valued process on $[0, T] \times \Omega$,

- $\mathcal{L}^{2}(\mathbb{R})=\left\{\eta: \mathcal{F}_{T}\right.$-measurable random $\mathbb{R}$ - valued variable s.t. $\left.\mathbb{E}\left[|\eta|^{2}\right]<\infty\right\}$,

- $\mathcal{H}^{2}(\mathbb{R})=\left\{v \in \mathcal{P}, \mathcal{F}_{\bullet}\right.$-predictable s.t. $\left.\mathbb{E}\left[\int_{0}^{T}|v(s)|^{2} \mathrm{~d} s\right]<\infty\right\}$,

- $\mathcal{S}^{2}(\mathbb{R})=\left\{Y \in \mathcal{P}, \mathcal{F}_{\bullet}\right.$-adapted, càdlàg and $\mathcal{F}_{T} \otimes \mathcal{B}([0, T])$ measurable s.t. $\left.\mathbb{E}\left[\sup _{0<t<T}\left|Y_{t}\right|^{2}\right]<\infty\right\}$,

- $\mathcal{S}_{i}^{2}=\left\{\left(k_{t}\right)_{0 \leq t \leq T}: \mathcal{F}_{\bullet}\right.$-adapted càdlàg increasing process, s.t. $k(0)=0$ and $\left.\mathbb{E}\left(K(T)^{2}\right)<\infty\right\}$,

- $L_{-T}^{2}(\mathbb{R})=\left\{z:[-T, 0] \rightarrow \mathbb{R}:\right.$ Borelian functions s.t $\int_{-T}^{0}|z(t)|^{2} \mathrm{~d} t<\infty$ a.s. $\}$,

- $L_{-T}^{\infty}(\mathbb{R})=\left\{y:[-T, 0] \rightarrow \mathbb{R}\right.$ : bounded, Borelian functions s.t $\sup _{t \in[-T, 0]}|y(t)|^{2}<\infty$ a.s $\}$.

Let $\mathcal{B}([-T, 0])$ the Borel sets of $[-T, 0]$ and $\lambda$ the Lebesgue measure on $([-T, 0], \mathcal{B}([-T, 0]))$. For some $\gamma>0$, $\mathcal{S}^{2}(\mathbb{R})$ and $\mathcal{H}^{2}(\mathbb{R})$ are endowed with the norms

$$
\|Y\|_{\mathcal{S}^{2}}^{2}=\mathbb{E}\left[\sup _{t \in[0, T]} \mathrm{e}^{\gamma t}|Y(t)|^{2}\right],
$$

and

$$
\|Z\|_{\mathcal{H}^{2}}^{2}=\mathbb{E}\left[\int_{0}^{T} \mathrm{e}^{\gamma t}|Z(t)|^{2} \mathrm{~d} t\right] .
$$

Now let $\xi$ be an $\mathcal{F}_{T}$-measurable $\mathbb{R}$-valued random variable and let us consider a function $g:[0, T] \times \Omega \times$ $L_{-T}^{\infty}(\mathbb{R}) \times L_{-T}^{2}(\mathbb{R}) \longrightarrow \mathbb{R}$ such that $t \rightarrow g\left(t, Y_{t}, Z_{t}\right)$ is an $\mathcal{F}_{\bullet}$-adapted process. Finally let $S:=\left\{S_{t}\right\}_{t \in[0, T]}$ be a càdlàg progressively measurable $\mathbb{R}$-valued process. 
By a solution to reflected BSDE with time delayed generator we mean a triple $(Y, Z, K):=\left(Y_{t}, Z_{t}, K_{t}\right)_{t \leq T}$ of processes with values in $\mathbb{R} \times \mathbb{R} \times \mathbb{R}_{+}$and which satisfies:

$$
\left\{\begin{array}{l}
\left(Y_{t}\right)_{0 \leq t \leq T} \in \mathcal{S}^{2}(\mathbb{R}),\left(Z_{t}\right)_{0 \leq t \leq T} \in \mathcal{H}^{2}(\mathbb{R}) \text { and }\left(K_{t}\right)_{0 \leq t \leq T} \in \mathcal{S}_{i}^{2} \\
Y(t)=\xi+\int_{t}^{T} g\left(s, Y_{s}, Z_{s}\right) \mathrm{d} s+K(T)-K(t)-\int_{t}^{T} Z(s) \mathrm{d} B(s), 0 \leq t \leq T \\
Y(t) \geq S(t), 0 \leq t \leq T, \\
(Y(t)-S(t)) \mathrm{d} K^{c}(t)=0, \text { and } \triangle_{t} Y:=Y(t)-Y\left(t^{-}\right)=-\left(S\left(t^{-}\right)-Y(t)\right)^{+}
\end{array}\right.
$$

where $K=K^{c}+K^{d}$, is the decomposition of $K$, with $K^{c}$ continuous and $K^{d}$ purely discontinuous. Here the generator $g$ at time $s \in[0, T]$ depends on the past values of the solution, denoted by $Y_{s}:=(Y(s+u))_{-T \leq u \leq 0}$ and $Z_{s}:=(Z(s+u))_{-T<u<0}$. We always set $Z(t)=0$ and $Y(t)=Y(0)$ for $t<0$.

It is worth noting that if the driver function $g$ does not depend on $(y, z)$, i.e., $\mathbb{P}$-a.s., $g(t, w, y, z)=g(t, w)$, this RBSDE exactly fits in the framework considered in Hamadène [12].

Remark 2.1. It follows from (2.1) that

$$
\triangle_{t} K^{d}=-\triangle_{t} Y=\left(S\left(t^{-}\right)-Y(t)\right)^{+}
$$

Therefore when $Y$ has a predictable jump we obligatorily have $S\left(t^{-}\right)=Y\left(t^{-}\right)$.

\section{EXISTENCE AND UNIQUENESS RESUlT}

In this section, we consider a delayed $\operatorname{RBSDE}(\xi, g, S)$ when generators, terminal condition and barrier process satisfy those assumptions:

(A1) The terminal value $\xi \in \mathcal{L}^{2}(\mathbb{R})$;

(A2) (i) The process $\{g(t, 0,0), 0 \leq t \leq T\}$ satisfies $\mathbb{E}\left(\int_{0}^{T}|g(t, 0,0)|^{2} \mathrm{~d} t\right)<\infty$,

(ii) $g(t, \omega, . .)=$.0 for $t<0$ and $\omega \in \Omega$.

(iii) $g$ is Lipschitz continuous in the sense that there exists a probability measure $\alpha$ on $([-T, 0], \mathcal{B}([-T, 0]))$ and a constant $C>0$ such that

$$
\left|g\left(t, y_{t}, z_{t}\right)-g\left(t, y_{t}^{\prime}, z_{t}^{\prime}\right)\right|^{2} \leq C\left(\int_{-T}^{0}\left|y(t+u)-y^{\prime}(t+u)\right|^{2} \alpha(\mathrm{d} u)+\int_{-T}^{0}\left|z(t+u)-z^{\prime}(t+u)\right|^{2} \alpha(\mathrm{d} u)\right),
$$

holds for $\mathbb{P} \times \lambda$-almost all $(\omega, t) \in \Omega \times[0, T]$ and for every $\left(y_{t}, z_{t}\right),\left(y_{t}^{\prime}, z_{t}^{\prime}\right) \in L_{-T}^{\infty}(\mathbb{R}) \times L_{-T}^{2}(\mathbb{R})$.

(A3) The obstacle process $S=(S(t), 0 \leq t \leq T)$ is an $\mathcal{F}_{\bullet}$-adapted càdlàg, real valued process, satisfying

$$
\mathbb{E}\left[\sup _{0 \leq t \leq T}\left(S_{t}^{+}\right)^{2}\right]<\infty, S_{t}^{+}=\max \left\{S_{t}, 0\right\}
$$

We now give a lemma concerning the generator.

Lemma 3.1. Suppose $(Y, Z, K)$ and $\left(Y^{\prime}, Z^{\prime}, K^{\prime}\right)$ are two solutions of the $R B S D E(g, \xi, S)$. Then

$$
\int_{t}^{T} \mathrm{e}^{\gamma s}\left|g\left(s, Y_{s}, Z_{s}\right)-g\left(s, Y_{s}^{\prime}, Z_{s}^{\prime}\right)\right|^{2} \mathrm{~d} s \leq C \tilde{\alpha}(\gamma)\left(T \sup _{0 \leq s \leq T} \mathrm{e}^{\gamma s}\left|Y(s)-Y^{\prime}(s)\right|^{2}+\int_{0}^{T} \mathrm{e}^{\gamma s}\left|Z(s)-Z^{\prime}(s)\right|^{2} \mathrm{~d} s\right),
$$

where $\tilde{\alpha}$ is

$$
\tilde{\alpha}(\gamma):=\int_{-T}^{0} \mathrm{e}^{-\gamma u} \alpha(\mathrm{d} u)
$$

In particular with $\gamma=0$

$$
\int_{t}^{T}\left|g\left(s, Y_{s}, Z_{s}\right)-g\left(s, Y_{s}^{\prime}, Z_{s}^{\prime}\right)\right|^{2} \mathrm{~d} s \leq C\left(T \sup _{0 \leq s \leq T}\left|Y(s)-Y^{\prime}(s)\right|^{2}+\int_{0}^{T}\left|Z(s)-Z^{\prime}(s)\right|^{2} \mathrm{~d} s\right) .
$$


Proof. Let $t \in[0, T]$. Since $g$ is Lipschitz continuous in the sense of (A2),

$$
\begin{aligned}
\int_{t}^{T} \mathrm{e}^{\gamma s}\left|g\left(s, Y_{s}, Z_{s}\right)-g\left(s, Y_{s}^{\prime}, Z_{s}^{\prime}\right)\right|^{2} \mathrm{~d} s \leq & C\left(\int_{t}^{T} \mathrm{e}^{\gamma s} \int_{-T}^{0}\left|Y(s+u)-Y^{\prime}(s+u)\right|^{2} \alpha(\mathrm{d} u) \mathrm{d} s\right. \\
& \left.+\int_{t}^{T} \mathrm{e}^{\gamma s} \int_{-T}^{0}\left|Z(s+u)-Z^{\prime}(s+u)\right|^{2} \alpha(\mathrm{d} u) \mathrm{d} s\right) .
\end{aligned}
$$

Applying Fubini's theorem and using the assumption that $Y(s)=Y(0)$ and $Z(s)$ vanishes for $s<0$, we obtain

$$
\begin{aligned}
\int_{t}^{T} \mathrm{e}^{\gamma s}\left|g\left(s, Y_{s}, Z_{s}\right)-g\left(s, Y_{s}^{\prime}, Z_{s}^{\prime}\right)\right|^{2} \mathrm{~d} s \leq & C\left(\int_{-T}^{0} \mathrm{e}^{-\gamma u} \int_{t}^{T} \mathrm{e}^{\gamma(s+u)}\left|Y(s+u)-Y^{\prime}(s+u)\right|^{2} \mathrm{~d} s\right) \alpha(\mathrm{d} u) \\
& \left.+\int_{-T}^{0} \mathrm{e}^{-\gamma u} \int_{t}^{T} \mathrm{e}^{\gamma(s+u)}\left|Z(s+u)-Z^{\prime}(s+u)\right|^{2} \mathrm{~d} s\right) \alpha(\mathrm{d} u) \\
= & C\left(\int_{-T}^{0} \mathrm{e}^{-\gamma u} \int_{(t+u) \vee 0}^{T+u} \mathrm{e}^{\gamma r}\left|Y(r)-Y^{\prime}(r)\right|^{2} \mathrm{~d} r \alpha(\mathrm{d} u)\right. \\
& \left.+\int_{-T}^{0} \mathrm{e}^{-\gamma u} \int_{(t+u) \vee 0}^{T+u} \mathrm{e}^{\gamma r}\left|Z(r)-Z^{\prime}(r)\right|^{2} \mathrm{~d} r \alpha(\mathrm{d} u)\right) \\
\leq & C\left(\int_{0}^{T} \mathrm{e}^{\gamma r}\left|Y(r)-Y^{\prime}(r)\right|^{2}\left(\int_{-T}^{0} \mathrm{e}^{-\gamma u} \alpha(\mathrm{d} u)\right) \mathrm{d} r\right. \\
& \left.+\int_{0}^{T} \mathrm{e}^{\gamma r}\left|Z(r)-Z^{\prime}(r)\right|^{2}\left(\int_{-T}^{0} \mathrm{e}^{-\gamma u} \alpha(\mathrm{d} u)\right) \mathrm{d} r\right) \\
\leq & C \tilde{\alpha}(\gamma)\left(T \sup _{0 \leq s \leq T} \mathrm{e}^{\gamma s}\left|Y(s)-Y^{\prime}(s)\right|^{2}+\int_{0}^{T} \mathrm{e}^{\gamma s}\left|Z(s)-Z^{\prime}(s)\right|^{2} \mathrm{~d} s\right) .
\end{aligned}
$$

Lemma 3.2 (cf. [12], inside the proof of Thm. 3.1). We keep the notations of Lemma 3.1 and we assume moreover that $\mathbb{P}$-a.s.

$$
\begin{aligned}
\int_{0}^{T}(Y(s)-S(s)) \mathrm{d} K^{c}(s)= & \int_{0}^{T}\left(Y^{\prime}(s)-S(s)\right) \mathrm{d} K^{\prime c}(s)=0 . \text { Then } \\
& \int_{] t, T]} \mathrm{e}^{\gamma s}\left(Y\left(s^{-}\right)-Y^{\prime}\left(s^{-}\right)\right)\left(\mathrm{d} K(s)-\mathrm{d} K^{\prime}(s)\right) \leq 0 .
\end{aligned}
$$

Our main result in this paper is:

Theorem 3.3. Let the assumptions (A1)-(A3) hold and let us assume that, the constants $T, C, C_{1}$ (where $C_{1}$ is a Burkholder-Davis-Gundy constant for martingale inequality) and the measure $\alpha$ are such that there exists $\gamma>0$ satisfying

$$
\left(4 C_{1}^{2}+3\right) C \tilde{\alpha}(\gamma) \max \{1, T\}<\gamma
$$

Then, there exists a unique solution $(Y, Z, K)$ in $\mathcal{S}^{2}(\mathbb{R}) \times \mathcal{H}^{2}(\mathbb{R}) \times \mathcal{S}_{i}^{2}$ of the delay reflected BSDE (2.1).

Proof. As in the non reflected case, the solution is obtained using the fixed point theorem. Let $\phi$ be the map from $\mathcal{D}_{\gamma}$ into itself, where $\mathcal{D}_{\gamma}$ is defined as the space of the progressively measurable processes $\left(Y_{t}, Z_{t}\right)_{0 \leq t \leq T}$, valued in $\mathbb{R} \times \mathbb{R}$ normed by

$$
\|(Y, Z)\|_{\gamma}^{2}=\mathbb{E}\left[\sup _{t \in[0, T]} \mathrm{e}^{\gamma t}|Y(t)|^{2}\right]+\mathbb{E}\left[\int_{0}^{T} \mathrm{e}^{\gamma t}|Z(t)|^{2} \mathrm{~d} t\right], \gamma>0 .
$$


Given $(U, V) \in \mathcal{D}_{\gamma}, \Phi(U, V)=(Y, Z)$ where $(Y, Z, K)$ is the solution of the reflected BSDE associated with $\left(g\left(t, U_{t}, V_{t}\right), \xi, S\right)$. Let $(\tilde{U}, \tilde{V})$ be another triple of $\mathcal{D}_{\gamma}$ and $\Phi(\tilde{U}, \tilde{V})=(\tilde{Y}, \tilde{Z})$. Applying Itô's formula to $\mathrm{e}^{\gamma t}\left(Y_{t}-\tilde{Y}_{t}\right)^{2}$ yields to:

$$
\begin{aligned}
\mathrm{e}^{\gamma t}(Y(t) & -\tilde{Y}(t))^{2}+\gamma \int_{t}^{T} \mathrm{e}^{\gamma s}(Y(s)-\tilde{Y}(s))^{2} \mathrm{~d} s+\int_{t}^{T} \mathrm{e}^{\gamma s}|Z(s)-\tilde{Z}(s)|^{2} \mathrm{~d} s+\sum_{t \leq s \leq T} \mathrm{e}^{\gamma s}\left(\Delta Y_{s}-\Delta \tilde{Y}_{s}\right)^{2} \\
= & \left(M_{T}-M_{t}\right)+2 \int_{t}^{T} \mathrm{e}^{\gamma s}\left(Y\left(s^{-}\right)-\tilde{Y}\left(s^{-}\right)\right)\left(g\left(s, U_{s}, V_{s}\right)-g\left(s, \tilde{U}_{s}, \tilde{V}_{s}\right)\right) \mathrm{d} s \\
& +2 \int_{t}^{T} \mathrm{e}^{\gamma s}\left(Y\left(s^{-}\right)-\tilde{Y}\left(s^{-}\right)\right)(d K(s)-\mathrm{d} \tilde{K}(s)),
\end{aligned}
$$

where $M_{t}=-2 \int_{0}^{t} \mathrm{e}^{\gamma s}\left(Y\left(s^{-}\right)-\tilde{Y}\left(s^{-}\right)\right)(Z(s)-\tilde{Z}(s)) \mathrm{d} B(s)$ is a uniformly integrable martingale. Using the elementary inequality $2 a b \leq \epsilon a^{2}+\frac{1}{\epsilon} b^{2}$, we get

$$
\begin{aligned}
\mathrm{e}^{\gamma t}(Y(t) & -\tilde{Y}(t))^{2}+\gamma \int_{t}^{T} \mathrm{e}^{\gamma s}(Y(s)-\tilde{Y}(s))^{2} \mathrm{~d} s+\int_{t}^{T} \mathrm{e}^{\gamma s}|Z(s)-\tilde{Z}(s)|^{2} \mathrm{~d} s+\sum_{t \leq s \leq T} \mathrm{e}^{\gamma s}\left(\Delta Y_{s}-\Delta \tilde{Y}_{s}\right)^{2} \\
\leq & \left(M_{T}-M_{t}\right)+\int_{t}^{T} \mathrm{e}^{\gamma s}\left\{\epsilon|Y(s)-\tilde{Y}(s)|^{2}+\frac{1}{\epsilon}\left|g\left(s, U_{s}, V_{s}\right)-g\left(s, \tilde{U}_{s}, \tilde{V}_{s}\right)\right|^{2}\right\} \mathrm{d} s \\
& +2 \int_{t}^{T} \mathrm{e}^{\gamma s}\left(Y\left(s^{-}\right)-\tilde{Y}\left(s^{-}\right)\right)(\mathrm{d} K(s)-\mathrm{d} \tilde{K}(s)) .
\end{aligned}
$$

Since $g$ satisfies (A2), Lemma 3.1 yields

$$
\int_{t}^{T} \mathrm{e}^{\gamma s}\left|g\left(s, U_{s}, V_{s}\right)-g\left(s, \tilde{U}_{s}, \tilde{V}_{s}\right)\right|^{2} \mathrm{~d} s \leq C \tilde{\alpha}(\gamma)\left(T \sup _{0 \leq s \leq T} \mathrm{e}^{\gamma s}|U(s)-\tilde{U}(s)|^{2}+\int_{0}^{T} \mathrm{e}^{\gamma s}|V(s)-\tilde{V}(s)|^{2} \mathrm{~d} s\right) .
$$

Henceforth,

$$
\begin{aligned}
\mathrm{e}^{\gamma t}(Y(t)-\tilde{Y}(t))^{2}+(\gamma-\epsilon) \int_{t}^{T} \mathrm{e}^{\gamma s}(Y(s)-\tilde{Y}(s))^{2} \mathrm{~d} s+\int_{t}^{T} \mathrm{e}^{\gamma s}|Z(s)-\tilde{Z}(s)|^{2} \mathrm{~d} s \\
\leq\left(M_{T}-M_{t}\right)+2 \int_{t}^{T} \mathrm{e}^{\gamma s}\left(Y\left(s^{-}\right)-\tilde{Y}\left(s^{-}\right)\right)(\mathrm{d} K(s)-\mathrm{d} \tilde{K}(s)) \\
+\frac{C \tilde{\alpha}(\gamma)}{\epsilon}\left(T \sup _{0 \leq s \leq T} \mathrm{e}^{\gamma s}|U(s)-\tilde{U}(s)|^{2}+\int_{0}^{T} \mathrm{e}^{\gamma s}|V(s)-\tilde{V}(s)|^{2} \mathrm{~d} s\right) .
\end{aligned}
$$

So by the Lemma 3.2 , it follows

$$
\begin{aligned}
& \mathrm{e}^{\gamma t}(Y(t)-\tilde{Y}(t))^{2}+(\gamma-\epsilon) \int_{t}^{T} \mathrm{e}^{\gamma s}(Y(s)-\tilde{Y}(s))^{2} \mathrm{~d} s+\int_{t}^{T} \mathrm{e}^{\gamma s}|Z(s)-\tilde{Z}(s)|^{2} \mathrm{~d} s \\
& \quad \leq\left(M_{T}-M_{t}\right)+\frac{C \tilde{\alpha}(\gamma)}{\epsilon}\left(T \sup _{0 \leq s \leq T} \mathrm{e}^{\gamma s}|U(s)-\tilde{U}(s)|^{2}+\int_{0}^{T} \mathrm{e}^{\gamma s}|V(s)-\tilde{V}(s)|^{2} \mathrm{~d} s\right) .
\end{aligned}
$$

By hypothesis we can choose $\epsilon \in]\left(4 C_{1}^{2}+3\right) C \tilde{\alpha}(\gamma) \max \{1, T\}, \gamma[$, thus

$$
\begin{aligned}
& \mathrm{e}^{\gamma t}(Y(t)-\tilde{Y}(t))^{2}+\int_{t}^{T} \mathrm{e}^{\gamma s}|Z(s)-\tilde{Z}(s)|^{2} \mathrm{~d} s \\
& \quad \leq\left(M_{T}-M_{t}\right)+\frac{C \tilde{\alpha}(\gamma)}{\epsilon}\left(T \sup _{0 \leq s \leq T} \mathrm{e}^{\gamma s}|U(s)-\tilde{U}(s)|^{2}+\int_{0}^{T} \mathrm{e}^{\gamma s}|V(s)-\tilde{V}(s)|^{2} \mathrm{~d} s\right) .
\end{aligned}
$$


This implies that

$\mathbb{E}\left[\int_{t}^{T} \mathrm{e}^{\gamma s}|Z(s)-\tilde{Z}(s)|^{2} \mathrm{~d} s\right] \leq \frac{C \tilde{\alpha}(\gamma)}{\epsilon} \max \{1, T\} \mathbb{E}\left[\sup _{0 \leq s \leq T} \mathrm{e}^{\gamma s}|U(s)-\tilde{U}(s)|^{2}+\int_{0}^{T} \mathrm{e}^{\gamma s}|V(s)-\tilde{V}(s)|^{2} \mathrm{~d} s\right]$

Next going back to (3.6) taking the supremum and then expectation, we get

$$
\begin{aligned}
& \mathbb{E}\left[\sup _{0 \leq t \leq T} \mathrm{e}^{\gamma t}(Y(t)-\tilde{Y}(t))^{2}\right] \leq \mathbb{E}\left[\sup _{0 \leq t \leq T}\left(M_{T}-M_{t}\right)\right] \\
& +\frac{C \tilde{\alpha}(\gamma)}{\epsilon} \max \{1, T\} \mathbb{E}\left[\sup _{0 \leq s \leq T} \mathrm{e}^{\gamma s}|U(s)-\tilde{U}(s)|^{2}+\int_{0}^{T} \mathrm{e}^{\gamma s}|V(s)-\tilde{V}(s)|^{2} \mathrm{~d} s\right]
\end{aligned}
$$

Using Burkholder-Davis-Gundy's inequality,

$$
\begin{aligned}
\mathbb{E}\left[\sup _{t \in[0, T]}\right. & \left.\left(M_{T}-M_{t}\right)\right]=\mathbb{E}\left[\sup _{t \in[0, T]}-2 \int_{t}^{T} \mathrm{e}^{\gamma s}(Y(s)-\tilde{Y}(s))(Z(s)-\tilde{Z}(s)) \mathrm{d} B(s)\right] \\
& \left.\leq 2 \mathbb{E}\left[\sup _{t \in[0, T]} \mid \int_{t}^{T} \mathrm{e}^{\gamma s}(Y(s)-\tilde{Y}(s))(Z(s)-\tilde{Z}(s)) \mathrm{d} B(s)\right]\right] \\
& \leq 2 C_{1} \mathbb{E}\left[\left(\int_{0}^{T} \mathrm{e}^{2 \gamma s}|Y(s)-\tilde{Y}(s)|^{2}|Z(s)-\tilde{Z}(s)|^{2} \mathrm{~d} s\right)^{1 / 2}\right] \\
& \leq \mathbb{E}\left[2\left(\sup _{t \in[0, T]} \mathrm{e}^{\gamma t / 2}|Y(t)-\tilde{Y}(t)|\right)\left(C_{1}^{2} \int_{0}^{T} \mathrm{e}^{\gamma s}|Z(s)-\tilde{Z}(s)|^{2} \mathrm{~d} s\right)^{1 / 2}\right] \\
& \leq \frac{1}{2} \mathbb{E}\left[\sup _{t \in[0, T]} \mathrm{e}^{\gamma t}|Y(t)-\tilde{Y}(t)|^{2}\right]+2 C_{1}^{2} \mathbb{E}\left[\int_{0}^{T} \mathrm{e}^{\gamma s}|Z(s)-\tilde{Z}(s)|^{2} \mathrm{~d} s\right] .
\end{aligned}
$$

Plugging this last inequality in (3.8), we obtain

$$
\begin{aligned}
\mathbb{E}\left[\sup _{t \in[0, T]} \mathrm{e}^{\gamma t}(Y(t)-\tilde{Y}(t))^{2}\right] \leq & \frac{C \tilde{\alpha}(\gamma)}{\epsilon} \max \{1, T\} \mathbb{E}\left[\sup _{s \in[0, T]} \mathrm{e}^{\gamma s}|U(s)-\tilde{U}(s)|^{2}+\int_{0}^{T} \mathrm{e}^{\gamma s}|V(s)-\tilde{V}(s)|^{2} \mathrm{~d} s\right] \\
& +\frac{1}{2} \mathbb{E}\left[\sup _{t \in[0, T]} \mathrm{e}^{\gamma t}|Y(t)-\tilde{Y}(t)|^{2}\right]+2 C_{1}^{2} \mathbb{E}\left[\int_{0}^{T} \mathrm{e}^{\gamma s}|Z(s)-\tilde{Z}(s)|^{2} \mathrm{~d} s\right]
\end{aligned}
$$

Hence,

$$
\begin{aligned}
& \mathbb{E}\left[\sup _{t \in[0, T]} \mathrm{e}^{\gamma t}(Y(t)-\tilde{Y}(t))^{2}\right]+\mathbb{E}\left[\int_{0}^{T} \mathrm{e}^{\gamma s}|Z(s)-\tilde{Z}(s)|^{2} \mathrm{~d} s\right] \\
& \leq \frac{2 C \tilde{\alpha}(\gamma)}{\epsilon} \max \{1, T\} \mathbb{E}\left[\sup _{s \in[0, T]} \mathrm{e}^{\gamma s}|U(s)-\tilde{U}(s)|^{2}+\int_{0}^{T} \mathrm{e}^{\gamma s}|V(s)-\tilde{V}(s)|^{2} \mathrm{~d} s\right] \\
& \quad+\left(4 C_{1}^{2}+1\right) \mathbb{E}\left[\int_{0}^{T} \mathrm{e}^{\gamma s}|Z(s)-\tilde{Z}(s)|^{2} \mathrm{~d} s\right]
\end{aligned}
$$


and then by (3.7) we have

$$
\begin{aligned}
& \mathbb{E}\left[\sup _{t \in[0, T]} \mathrm{e}^{\gamma t}(Y(t)-\tilde{Y}(t))^{2}\right]+\mathbb{E}\left[\int_{0}^{T} \mathrm{e}^{\gamma s}|Z(s)-\tilde{Z}(s)|^{2} \mathrm{~d} s\right] \\
& \leq\left(4 C_{1}^{2}+3\right) \frac{C \tilde{\alpha}(\gamma)}{\epsilon} \max \{1, T\} \mathbb{E}\left[\sup _{s \in[0, T]} \mathrm{e}^{\gamma s}|U(s)-\tilde{U}(s)|^{2}+\int_{0}^{T} \mathrm{e}^{\gamma s}|V(s)-\tilde{V}(s)|^{2} \mathrm{~d} s\right] .
\end{aligned}
$$

Since $\epsilon$ is such that $\left(4 C_{1}^{2}+3\right) \frac{C \tilde{\alpha}(\gamma)}{\epsilon} \max \{1, T\}<1$, the map $\Phi$ is a contraction on $\mathcal{D}_{\gamma}$ with the norm (3.5). Henceforth there exists a pair $(Y, Z) \in \mathcal{D}_{\gamma}$ such that $\Phi(Y, Z)=(Y, Z)$ which, with $K$, is the unique solution of the time delayed reflected BSDE associated with $(g, \xi, S)$.

Remark 3.4. Condition (3.4) gives that the unique solution of (2.1) exists under (A1)-(A3) provided that

$$
\frac{\tilde{\alpha}(\gamma)}{\gamma}<\frac{1}{\left(4 C_{1}^{2}+3\right) C \max \{1, T\}}
$$

This implies that existence and uniqueness hold only if the Lipschitz's constant $C>0$ or the terminal time $T>0$ are sufficiently small.

An example is as follows.

Example 3.5. Consider the case $[0, T]=[0,1], C=\frac{1}{4\left(4 C_{1}^{2}+3\right)}, g\left(t, y_{t}, z_{t}\right)=\int_{0}^{t}|y(s)| \mathrm{d} s$ and $\alpha(\mathrm{d} s)=\mathrm{d} s$. It is clear that $\tilde{\alpha}(\gamma)=\frac{\mathrm{e}^{\gamma}-1}{\gamma}$. Obviously $\frac{1}{4}\left(\mathrm{e}^{\gamma}-1\right)-\gamma^{2}<0$ on the interval $[0.3,4.33]$. So, for any $\gamma \in[0.3,4.33]$ we have $\frac{\tilde{\alpha}(\gamma)}{\gamma}<\frac{1}{\left(4 C_{1}^{2}+3\right) C \max \{1, T\}}$.

But if $T$ is great enough, there exist cases where the solution does not exist. For instance, let $\xi$ such that $\mathbb{E}[\xi]>0$ and the obstacle $S(t)=a>0$. In such case,

$$
\mathbb{E}[\xi+K(T)]=Y(0)-\int_{0}^{T} \int_{0}^{s} \mathbb{E}(Y(u)) \mathrm{d} u \mathrm{~d} s .
$$

Thus, if $T^{2}>\frac{2 Y(0)}{a}$ there is a contradiction and therefore the delayed RBSDE does not have any solution.

Actually condition (3.4) is strong. For illustration let us provide another example (inspired from the one of Delong and Imkeller [7]) where (3.4) is not satisfied and there is no solution.

Example 3.6. Let $g\left(s, Y_{s}, Z_{s}\right)=C Y(s-T)$, where $C$ is the Lipschitz constant of the time delayed generators. Let $\alpha(\mathrm{d} s)=\delta_{-T}(\mathrm{~d} s)$ and suppose that $\mathbb{E}(\xi)>0$. Assume that there exists a solution $(Y(t), Z(t), K(t))_{0 \leq t \leq T}$ of the following delayed RBSDE

$$
\left\{\begin{array}{l}
Y(t)=\xi+C \int_{t}^{T} Y(s-T) \mathrm{d} s+K(T)-K(t)-\int_{t}^{T} Z(s) \mathrm{d} B(s), \\
Y(t) \geq S(t), \quad 0 \leq t \leq T, \\
(Y(t)-S(t)) \mathrm{d} K^{c}(t)=0 \quad \text { and } \quad \Delta_{t} Y:=Y(t)-Y\left(t^{-}\right)=-\left(S\left(t^{-}\right)-Y(t)\right)^{+} .
\end{array}\right.
$$

For any barrier $S$, the solution satisfies

$$
Y(t)=\xi+C(T-t) Y(0)+K(T)-K(t)-\int_{t}^{T} Z(s) \mathrm{d} B(s)
$$

Putting $t=0$ and taking expectation yields

$$
\mathbb{E}[\xi+K(T)]=(1-T C) Y(0) .
$$


In the case when $T C=1$, there is a contradiction since

$$
\mathbb{E}[\xi+K(T)]>0 .
$$

Moreover, clearly there is no $\gamma$ such that (3.4) could be satisfied with such a choice of $T$.

In general we do not have a comparison theorem for solutions of BSDEs with time delayed generators, whether they are reflected or not (see Delong-Imkeller [7]). However in some specific cases, when $Y$ and the control process has some features and especially when they stay away from 0 and $\infty$, we actually have a comparison result.

\section{Comparison Result of BSDE With CONTINuOus Barrier}

Let us recall the existence result for the solutions of the following reflected BSDEs with continuous barrier with time delayed generators

$$
\begin{aligned}
& Y(t)=\xi+\int_{t}^{T} g\left(s, Y_{s}, Z_{s}\right) \mathrm{d} s+K(T)-K(t)-\int_{t}^{T} Z(s) \mathrm{d} B(s), 0 \leq t \leq T \\
& Y(t) \geq S(t), 0 \leq t \leq T \quad \text { and } \quad(Y(t)-S(t)) \mathrm{d} K(t)=0 .
\end{aligned}
$$

Instead of (A3) we assume

(A3') the obstacle $\{S(t), 0 \leq t \leq T\}$, is a continuous progressively measurable real-valued process satisfying $\mathbb{E}\left[\sup _{0 \leq t \leq T}\left(S^{+}(t)\right)^{2}\right]<\infty$

Theorem 4.1 (cf. Zhou and Ren [18]). Assume assumptions (A1), (A2) and (A3') hold. If there exists a positive constant $\gamma$ satisfying

$$
2 C \tilde{\alpha}(\gamma)<\gamma,
$$

then the RBSDE with time delayed generator (4.1) has a unique solution $(Y(t), Z(t), K(t)), 0 \leq t \leq T$.

The following result allows us to locally compare the components $Y$ 's on a stochastic interval $\left[0, \tau_{t, n}\right]$. Namely we have:

Theorem 4.2. Let us consider the solutions $\left(Y^{1}, Z^{1}, K^{1}\right)$ and $\left(Y^{2}, Z^{2}, K^{2}\right)$ of two delayed reflected BSDEs associated with parameters $\left(g^{1}, \xi^{1}, S^{1}\right)$ and $\left(g^{2}, \xi^{2}, S^{2}\right)$ satisfying assumptions (A1) and (A3'). For $n \in \mathbb{N}$ and $0 \leq t \leq T$ define the stopping times

$$
\begin{gathered}
\tau_{n}:=\inf \left\{s \geq 0,\left|Y^{1}(s)-Y^{2}(s)\right| \wedge\left|Z^{1}(s)-Z^{2}(s)\right| \leq \frac{1}{n}\right. \\
\text { or } \left.\left|Y^{1}(s)-Y^{2}(s)\right| \vee\left|Z^{1}(s)-Z^{2}(s)\right| \geq n\right\} \wedge T, \\
\tau_{t}^{i}=\inf \left\{s \geq t, Y^{i}(s)=S^{i}(s)\right\} \wedge \tau_{n}, i=1,2 \text { and } \tau_{t, n}=\tau_{t}^{1} \wedge \tau_{t}^{2} .
\end{gathered}
$$

Assume (A2) holds for only the coefficient $g^{1}$ and that

$$
\begin{aligned}
& Y^{1}\left(\tau_{t, n}\right) \leq Y^{2}\left(\tau_{t, n}\right), \mathbb{P}-a . s \\
& g^{1}\left(t, Y_{t}^{1}, Z_{t}^{1}\right) \leq g^{2}\left(t, Y_{t}^{1}, Z_{t}^{1}\right), \mathbb{P} \times \lambda-a . s, \\
& S^{1}(t) \leq S^{2}(t), \mathbb{P} \times \lambda-\text { a.s. }
\end{aligned}
$$

Then $Y^{1}(t) \leq Y^{2}(t)$, for $t \in\left[0, \tau_{t, n}\right]$, a.s.

Remark 4.3. The hypothesis " $Y^{1}\left(\tau_{t, n}\right) \leq Y^{2}\left(\tau_{t, n}\right) \mathbb{P}$-a.s" could be interpreted as following: if at the first reflection time of one or the other solution $Y^{i}, Y^{1} \leq Y^{2}$, then it is true before this reflection time. 
Proof. The key is to use the sequence of stopping times $\left(\tau_{n}\right)$ as in Delong-Imkeller [7].

Denote $(\delta Y(t), \delta Z(t))=\left(Y^{1}(t)-Y^{2}(t), Z^{1}(t)-Z^{2}(t)\right)$ and $\delta g^{1}\left(t, Y_{t}^{2}, Z_{t}^{2}\right)=g^{1}\left(t, Y_{t}^{2}, Z_{t}^{2}\right)-g^{2}\left(t, Y_{t}^{2}, Z_{t}^{2}\right)$. Let us then define the following adapted processes

$$
\Delta_{y} g^{1}(t)=\frac{g^{1}\left(t, Y_{t}^{1}, Z_{t}^{1}\right)-g^{1}\left(t, Y_{t}^{2}, Z_{t}^{1}\right)}{\delta Y(t)} \quad \text { and } \quad \Delta_{z} g^{1}(t)=\frac{g^{1}\left(t, Y_{t}^{2}, Z_{t}^{1}\right)-g^{1}\left(t, Y_{t}^{2}, Z_{t}^{2}\right)}{\delta Z(t)} .
$$

By the definition of the reflected BSDE $(4.1),(\delta Y, \delta Z)$ satisfies

$$
-\mathrm{d} \delta Y(s)=\left(\Delta_{y} g^{1}(s) \delta Y(s)+\Delta_{z} g^{1}(s) \delta Z(s)+\delta g^{1}\left(s, Y_{s}^{2}, Z_{s}^{2}\right)\right) \mathrm{d} s+\mathrm{d} \delta K(s)-\delta Z(s) \mathrm{d} W(s) .
$$

Let us set $R(t, s)=\exp \left(\int_{t}^{s} \Delta_{y} g^{1}(r) \mathrm{d} r\right)$, then applying Itô's formula to process $s \mapsto R(t, s) \delta Y(s)$, we get

$$
\delta Y\left(\tau_{t, n}\right) R\left(t, \tau_{t, n}\right)-\delta Y(t) R(t, t)=\int_{t}^{\tau_{t, n}} R(t, s) \mathrm{d} \delta Y(s)+\int_{t}^{\tau_{t, n}} \delta Y(s) \mathrm{d}(R(t, s)) .
$$

Hence,

$$
\begin{aligned}
\delta Y(t) & =\delta Y\left(\tau_{t, n}\right) R\left(t, \tau_{t, n}\right)-\int_{t}^{\tau_{t, n}} R(t, s) \mathrm{d} \delta Y(s)-\int_{t}^{\tau_{t, n}} \mathrm{~d}(R(t, s)) \delta Y(s) \\
& =\delta Y\left(\tau_{t, n}\right) R\left(t, \tau_{t, n}\right)+\int_{t}^{\tau_{t, n}} R(t, s)\left\{\left(\Delta_{z} g^{1}(s) \delta Z(s)+\delta g^{1}\left(s, Y_{s}^{2}, Z_{s}^{2}\right)\right) \mathrm{d} s+\mathrm{d} \delta K(s)-\delta Z(s) \mathrm{d} W(s)\right\}
\end{aligned}
$$

The condition $\int_{0}^{T}\left(Y^{i}(t)-S^{i}(t)\right) \mathrm{d} K^{i}(t)=0$ and the continuity of $K^{i}($.$) imply that$

$$
K^{1}\left(\tau_{t}^{1}\right)-K^{1}(t)=0, \quad K^{2}\left(\tau_{t}^{2}\right)-K^{2}(t)=0 .
$$

Therefore,

$$
\delta Y(t)=\delta Y\left(\tau_{t, n}\right) R\left(t, \tau_{t, n}\right)+\int_{t}^{\tau_{t, n}} \delta g^{1}\left(s, Y_{s}^{2}, Z_{s}^{2}\right) R(t, s) \mathrm{d} s-\int_{t}^{\tau_{t, n}} \delta Z(s) R(t, s) \mathrm{d} \tilde{W}(s), \quad 0 \leq t \leq \tau_{t, n},
$$

where $\tilde{W}(s)=W(s)-\int_{0}^{s} \Delta_{z} g^{1}(r) R(t, r) \mathrm{d} r$ is a Brownian motion under the probability $\tilde{\mathbb{P}}$ defined as:

$$
\left.\frac{\mathrm{d} \tilde{\mathbb{P}}}{\mathrm{dP}}\right|_{\mathcal{F}_{\tau_{t, n}}}=\exp \left(\int_{0}^{\tau_{t, n}} \Delta_{z} g^{1}(s) \mathrm{d} W(s)-\frac{1}{2} \int_{0}^{\tau_{t, n}}\left(\Delta_{z} g^{1}(s)\right)^{2} \mathrm{~d} s\right) .
$$

Let us observe that $\delta Z \in \mathcal{H}^{2}(\mathbb{R})$, the density $\left.\frac{\mathrm{d} \tilde{\mathbb{P}}}{\mathrm{d} \mathbb{P}}\right|_{\mathcal{F}_{\tau_{t, n}}}$ is square integrable under the measure $\mathbb{P}$, since $t \rightarrow \Delta_{z} g^{1}(t)$ is a.s. uniformly bounded up to time $\tau_{t, n} \leq \tau_{n}$. Thus, using Cauchy-Schwarz's inequality, we obtain

$$
\mathbb{E}\left[\left(\int_{0}^{\tau_{t, n}} R(s)^{2}|\delta Z(s)|^{2} \mathrm{~d} s\right)^{1 / 2}\right]<+\infty .
$$

Therefore taking the $\mathcal{F}_{t}$ conditional expectation in (4.2) with respect to $\tilde{\mathbb{P}}$ yields to

$$
\delta Y(t)=\tilde{\mathbb{E}}\left[\delta Y\left(\tau_{t, n}\right) R\left(t, \tau_{t, n}\right)+\int_{t}^{\tau_{t, n}} \delta g^{1}\left(s, Y_{s}^{2}, Z_{s}^{2}\right) R(t, s) \mathrm{d} s \mid \mathcal{F}_{t}\right], \quad 0 \leq t \leq \tau_{t, n} .
$$

By hypothesis $\delta Y\left(\tau_{t, n}\right) \leq 0$ and $\delta g^{1}\left(s, Y_{s}^{2}, Z_{s}^{2}\right) \leq 0$, so $\delta Y(t) \leq 0$.

Remark 4.4. This result concerns a specific case, but using a quite similar proof, another example could be provided: in case of $S^{2}$ discontinuous, but $\left(Y^{1}, Z^{1}\right)$ solution of a non reflected BSDE, under the hypothesis $Y^{1}\left(\tau_{n}\right) \leq Y^{2}\left(\tau_{n}\right)$, we have $Y^{1}(t) \leq Y^{2}(t)$, for $t \in\left[0, \tau_{n}\right]$, a.s.

Acknowledgements. Author would like to thank professor Monique Pontier for the helpful suggestions and comments. 


\section{REFERENCES}

[1] G. Barles, R. Buchdahn and E. Pardoux, BSDE's and integral-partial differential equations. Stoch. Stoch. Rep. 60 (1997) 57-83.

[2] J.M. Bismut, Conjugate convex functions in optimal stochastic control. J. Math. Anal. Appl. 44 (1973) $384-404$.

[3] J. Cvitanic and I. Karatzas, Backward SDEs with reflection and Dynkin games. Ann. Probab. 24 (1996) $2024-2056$.

[4] C. Dellacherie and P.A. Meyer, Probabilités et Potentiel, Chapter V-VII. Hermann, Paris (1980).

[5] Ł. Delong, BSDEs with time-delayed generators of a moving average type with applications to pricing and utilities (2010). Preprint arXiv: 1008.3722.

[6] Ł. Delong, Applications of time-delayed backward stochastic differential equations to pricing, hedging and management of insurance and financial risks (2011). Preprint arXiv:1005.4417v3.

[7] Ł. Delong and P. Imkeller, Backward stochastic differential equations with time delayed generator - Results and counterexamples. Ann. Appl. Probab. 20 (2010) 1512-1536.

[8] Ł. Delong and P. Imkeller, On Malliavin's differentiability of time delayed BSDEs driven by Brownian motions and Poisson random measures. Stoch. Process. Appl. 120 (2010) 1748-1775.

[9] G. Dos Reis, A. Reveillac and J. Zhang, FBSDE with time delayed generators $-L^{p}$ solutions, differentiability, representation formulas and path regularity. Stoch. Process. Appl. 121 (2011) 2114-2150.

[10] N. El-Karoui, C. Kapoudjian, E. Pardoux, S. Peng and M.C. Quenez, Reflected solutions of backward SDE's and related obstacle problems for PDE's. Ann. Probab. 25 (1997) 702-737.

[11] N. El Karoui, S. Peng and M.C. Quenez, Backward stochastic differential equations in finance. Math. Finance 7 (1997) 1-71.

[12] S. Hamadène, Reflected BSDEs with discontinuous barrier and applications. Stoch. Stoch. Rep. 74 (2002) $571-596$.

[13] S. Hamadène, Mixed Zero-sum differential game and American game options. SIAM J. Control. Optim. (2006).

[14] S. Hamadène and J.-P. Lepeltier, Backward equations, stochastic control and zero-sum stochastic differential games. Stoch. Stoch. Rep. 54 221-231, 1995.

[15] E. Pardoux, BSDEs, weak convergence and homogenization of semilinear PDEs, edited by F. Clarke and R. Stern. Nonlin. Anal., Differ. Equ. Control. Kluwer Acad. Publi., Netherlands (1999) 503-549.

[16] E. Pardoux and S. Peng, Adapted solution of a backward stochastic differential equation. Syst. Control Lett. 14 (1990) 55-61.

[17] E. Pardoux and S. Peng, Backward stochastic differential equations and quasilinear parabolic partial differential equations, in: Stochastic Partial Differential Equations and their Applications, Charlotte, NC (1991). In vol. 176 of Lect. Notes Control Inform. Sci. Springer, Berlin (1992) 200-217.

[18] Q. Zhou and Y. Ren, Reflected backward stochastic differential equations with time delayed generators. Stat. Probab. Lett. 82 (2012) 979-990. 